\title{
Elicitable distortion risk measures: a concise proof
}

\author{
Ruodu Wang* and Johanna F. Ziegel ${ }^{\dagger}$
}

February 4, 2015

\begin{abstract}
Elicitability has recently been discussed as a desirable property for risk measures. Kou and Peng (2014) showed that an elicitable distortion risk measure is either a Value-at-Risk or the mean. We give a concise alternative proof of this result, and discuss the conflict between comonotonic additivity and elicitability.
\end{abstract}

Key-words: distortion risk measures; elicitability; Value-at-Risk.

\section{Distortion risk measures}

We consider a standard atomless probability space $(\Omega, \mathcal{F}, \mathbb{P})$, and denote by $D$ the set of distribution functions on $\mathbb{R}$. A law invariant risk measure $\rho$ is a mapping from $D_{\rho}$ to $[-\infty,+\infty]$, where $D_{\rho} \subset D$. Write

$$
H=\{h:[0,1] \rightarrow[0,1]: h \text { is non-decreasing, } h(0)=0 \text { and } h(1)=1\} .
$$

Definition 1.1. A distortion risk measure $\rho: D_{\rho} \rightarrow \mathbb{R}$ is defined by

$$
\rho(F)=\int_{-\infty}^{0}(h(1-F(x))-1) \mathrm{d} x+\int_{0}^{\infty} h(1-F(x)) \mathrm{d} x,
$$

*Corresponding author. Department of Statistics and Actuarial Science, University of Waterloo, Canada. Email: wang@uwaterloo.ca

$\dagger$ Department of Mathematics and Statistics, University of Bern, Switzerland.

Email: johanna.ziegel@stat.unibe.ch 
where $D_{\rho}$ is set of some $F \in D$ such that (1.1) is well-defined, and $h \in H$ is called the distortion function of $\rho$.

The two most popular risk measures used in practice, Value-at-Risk (VaR) and Expected Shortfall (ES), are both distortion risk measures; for a recent discussion on VaR and ES, see Embrechts et al. (2014). We refer to Wang et al. (1997), Acerbi (2002), Kusuoka (2001) and Kou and Peng (2014) for more details and examples of distortion risk measures. Distortion risk measures are closely related to L-statistics (linear combinations of rank statistics, introduced for robust estimation); see Chapter 3 of Huber and Ronchetti (2009).

\section{Elicitability}

Elicitability was originally introduced as a property of set-valued functions. For consistency, we consider $\rho: D_{\rho} \rightarrow 2^{\mathbb{R}}$ as set-valued functions. This includes, for example, the case of quantiles, which may be an interval. In most cases, each value of $\rho$ is a set with exactly one element as in Section 1, and we simply treat them as mappings to $\mathbb{R}$.

Definition 2.1. A scoring function $S: \mathbb{R} \times \mathbb{R} \rightarrow[0, \infty)$ is called consistent for $\rho$ with respect to $D_{\rho}$, if

$$
\mathbb{E}(S(t, X)) \leqslant \mathbb{E}(S(x, X))
$$

for all $t \in \rho(F)$ and all $x \in \mathbb{R}$, where $X$ is a random variable with distribution $F \in D_{\rho}$. We speak of strict consistency of $S$ if equality in (2.1) implies $x \in \rho(X)$.

The functional $\rho$ is elicitable (with respect to $D_{\rho}$ ) if there exists a strictly consistent scoring function for it.

Roughly speaking, the forecasting of an elicitable risk measure can be evaluated using a score function, whereas there is no clear criterion to evaluate the forecasting of a non-elicitable risk measure. Arguments for the desirability of 
elicitability for risk management and other statistical sciences can be found in Gneiting (2011) and Ziegel (2015).

\section{Elicitable distortion risk measures}

A necessary condition for a functional to to be elicitable are convex level sets, that is, if $t \in \rho(F) \cap \rho(G)$, then

$$
t \in \rho(\lambda F+(1-\lambda) G)
$$

whenever $\lambda F+(1-\lambda) G \in D_{\rho}$ for $\lambda \in[0,1]$; see Osband (1985). Developments on risk measures with convex level sets can be found in Weber (2006), Lambert (2012), Ziegel (2015), Bellini and Bignozzi (2014), Kou and Peng (2014) and Delbaen et al. (2014). The work of Steinwart et al. (2014) shows that convex level sets are also a sufficient criterion for elicitability under some weak regularity assumptions on $\rho$; see also Lambert (2012).

The work of Weber (2006) investigated monetary risk measures with convex level sets under some additional regularity assumptions in a context of dynamic consistency. Under his assumptions, convex level sets are necessary and sufficient for the risk measure to be a shortfall risk measure. In the case of convex risk measures, Delbaen et al. (2014) show that Weber's (2006) assumptions are equivalent to the weak compactness property. They extend his result by showing that all convex risk measures with convex level sets are necessarily generalized shortfall risk measures. Bellini and Bignozzi (2014) considered monetary elicitable risk measures based on the results of Weber (2006). They use a more restrictive definition of elicitability by imposing regularity conditions on the scoring function $S$, which in turn ensures that Weber's assumptions are satisfied. It was shown that (1) a monetary risk measure is elicitable only if it is a shortfall risk measure; (2) a convex risk measure is elicitable if and only if it is a convex shortfall; (3) a coherent risk measure is elicitable if and only if it is an expectile. In the case 
of coherent risk measures it is possible to show directly from the Kusuoka representation (Kusuoka, 2001), that the only coherent risk measures with convex level sets are expectiles; see Ziegel (2015). While it is possible to apply Weber's (2006) results to distortion risk measures, this requires unnecessary additional assumptions, which can be avoided by exploiting the structure of distortion risk measures directly.

The following result (Kou and Peng, 2014, Theorem A.1) characterizes distortion risk measures with convex level sets, which leads to a full characterization of elicitable distortion risk measures. We provide an alternative proof of this result, which is substantially shorter and less technical.

Theorem 3.1 (Kou and Peng (2014)). Let $D^{*}$ be the class of distributions with finite support and $\rho$ be a distortion risk measure with distortion function $h \in H$ as defined at (1.1) whose restriction to $D^{*}$ has convex level sets. Then $h$ is either the identity on $[0,1]$ or it is of the form

$$
h(x)= \begin{cases}0, & x \in[0, \alpha), \\ c, & x=\alpha, \\ 1, & x \in(\alpha, 1],\end{cases}
$$

for some $\alpha, c \in[0,1]$. If $\alpha=0$ or $\alpha=1$, then $c=0$ or $c=1$, respectively.

Proof. Let $0<x<y, \lambda \in[0,1]$. Then

$$
\rho\left((1-\lambda) \delta_{x}+\lambda \delta_{y}\right)=x+h(\lambda)(y-x)
$$

where $\delta_{x}$ is the Dirac measure at the point $x \in \mathbb{R}$. In particular, $\rho\left(\delta_{1}\right)=1$. Let $\lambda \in[0,1]$ such that $h(\lambda)>0$. All $0<x<y$ such that $\rho\left((1-\lambda) \delta_{x}+\lambda \delta_{y}\right)$ are characterized by the equation

$$
y=\left(1-\frac{1}{h(\lambda)}\right) x+\frac{1}{h(\lambda)}
$$

In order to obtain $x<y$, we need to choose $x<1$, which then implies $y>1$. 
Convexity of level sets on $D^{*}$ now implies for all $p \in[0,1], 0<x<1<y$ chosen as described before, that

$$
\begin{aligned}
1 & =\rho\left(p\left((1-\lambda) \delta_{x}+\lambda \delta_{y}\right)+(1-p) \delta_{1}\right) \\
& =x+h(1-p(1-\lambda))(1-x)+h(\lambda p)\left(1-\frac{1}{h(\lambda)}\right)(1-x),
\end{aligned}
$$

hence

$$
h(\lambda)=h(\lambda) h(1-(1-\lambda) p)+(1-h(\lambda)) h(\lambda p), \quad \lambda, p \in[0,1] .
$$

Let $\lambda_{0}:=\inf \{t \mid h(t)>0\}$. Assume that $\lambda_{0}>0$, and let $\lambda \in\left(\lambda_{0}, 1\right]$. Then, $h(\lambda)>0$ and for $p \in\left[0, p_{0}\right)$, we have $h(\lambda p)=0$. Equation (3.2) now implies that $h(t)=1$ for $t \in\left(1-p_{0}(1-\lambda), 1\right]=\left(1-\lambda_{0}(1-\lambda) / \lambda, 1\right]$. As this holds for all $\lambda \in\left(\lambda_{0}, 1\right]$, we obtain $h(t)=1$ for $t \in\left(\lambda_{0}, 1\right]$, hence $h$ is of the form (3.1).

Assume form now on that $\lambda_{0}=0$, i.e. $h>0$ on $(0,1]$. If there is $\lambda^{\prime} \in(0,1)$ such that $h\left(\lambda^{\prime}\right)=1$, pick $\lambda<\lambda^{\prime}$ and $p \in[0,1]$ such that $\lambda^{\prime}=1-p(1-\lambda)$. Then (3.2) implies that $h(\lambda)=1$, hence $h=1$ on $(0,1]$, and $h$ is of the form (3.1).

So suppose now that $0<h<1$ on $(0,1)$. Integrating (3.2) over $p \in[0,1]$ we obtain for $\lambda \in(0,1)$

$$
\begin{aligned}
h(\lambda) & =h(\lambda) \int_{0}^{1} h(1-(1-\lambda) p) d p+(1-h(\lambda)) \int_{0}^{1} h(\lambda p) d p \\
& =\frac{h(\lambda)}{1-\lambda} \int_{\lambda}^{1} h(x) d x+\frac{1-h(\lambda)}{\lambda} \int_{0}^{\lambda} h(x) d x \\
& =\frac{h(\lambda)}{1-\lambda}(g(1)-g(\lambda))+\frac{1-h(\lambda)}{\lambda} g(\lambda),
\end{aligned}
$$

where $g(\lambda)=\int_{0}^{\lambda} h(x) d x$. Or, equivalently

$$
h(\lambda)\left(\lambda-\frac{\lambda}{1-\lambda}(g(1)-g(\lambda))+\frac{1}{\lambda} g(\lambda)\right)=g(\lambda) .
$$

The function $g$ is continuous on $(0,1)$, hence (3.3) shows that also $h$ is continuous in $(0,1)$. But this implies in turn that $g$ is continuously differentiable in $(0,1)$. Because $h>0$, we obtain that $\lambda-\frac{\lambda}{1-\lambda}(g(1)-g(\lambda))+\frac{1}{\lambda} g(\lambda)>0$ on $(0,1)$. Hence,

$$
h(\lambda)=\frac{g(\lambda)}{\lambda-\frac{\lambda}{1-\lambda}(g(1)-g(\lambda))+\frac{1}{\lambda} g(\lambda)},
$$


which implies the continuous differentiability of $h$ on $(0,1)$.

Differentiating (3.2) with respect to $p$ yields

$$
-h(\lambda) h^{\prime}(1-p(1-\lambda))(1-\lambda)+(1-h(\lambda)) h^{\prime}(\lambda p) \lambda=0
$$

Let $H=\left\{\lambda \in(0,1): h^{\prime}(\lambda)>0\right\}$, obviously non-empty. We obtain, by putting $p=1$, that $h(\lambda)=\lambda$ for $\lambda \in H$. As $h^{\prime}$ is continuous, $H$ is an open set; therefore $h^{\prime}(\lambda)=1$ for $\lambda \in H$, which along with the continuity of $h^{\prime}$ further implies $H=$ $(0,1)$, that is, $h(\lambda)=\lambda$ on $(0,1)$.

Theorem 3.1 implies that an elicitable distortion risk measure with respect to any set containing $D^{*}$ has to be either a quantile or the mean. Since distortion risk measures are continuous with respect to the Wasserstein distance and are translation-invariant, one can easily see that if a distortion risk measure has convex level sets on all bounded absolutely continuous distributions, then it has to have convex level sets on $D^{*}$.

If $h$ is the identity on $[0,1]$, then $\rho$ is the mean, which is elicitable with respect to the class of all integrable probability distributions; see Savage (1971) and Banerjee et al. (2005). If $h=c \mathbb{1}_{\{\alpha\}}+\mathbb{1}_{(\alpha, 1]}$, then $\rho$ is an $\alpha$-quantile, which is elicitable to the class of all absolutely continuous probability distributions; see Thomson (1979) and Gneiting (2011).

Strictly speaking, a left-quantile (or right-quantile or a convex combination of the two) is not elicitable with respect to $D^{*}$ despite its convex level sets. However, we do not believe that this is a problem in applications where quantile forecasts are to be evaluated and compared because in the case of distributions with positive density functions on their support, quantiles are unique.

In summary, for all practical purposes Value-at-Risk (quantiles) and the mean are elicitable distortion risk measures and they are the only ones. This characterization was given in Kou and Peng (2014, Theorem 2.1) in a slightly different form (elicitability is treated for single-valued functions in the latter paper; there is no essential difference). 
Remark 3.1. We observe from the proof that it is already sufficient to choose $D^{*}$ as the class of three-point distributions. Hence, any distortion risk measures except for the mean and Value-at-Risk are not elicitable with respect to bounded discrete distributions.

\section{Discussion}

Roughly speaking, there is a fundamental conflict between distortion and elicitability. This is due to the fact that comonotonic additivity, which is the essential property for distortion risk measures, requires linearity on the inverse distribution functions, that is,

$$
\rho\left(\lambda F^{-1}(U)+(1-\lambda) G^{-1}(U)\right)=\lambda \rho\left(F^{-1}(U)\right)+(1-\lambda) \rho\left(G^{-1}(U)\right), \quad \lambda>0,
$$

where $U$ is a uniform random variable on $[0,1]$ and $F$ and $G$ are two distribution functions in $D_{\rho}$. On the other hand, elicitability requires convex level sets on the level of distribution functions, that is,

$$
\rho(\lambda F+(1-\lambda) G)=\lambda \rho(F)+(1-\lambda) \rho(G), \quad \lambda \in(0,1),
$$

where $F$ and $G$ are two distributions functions in $D_{\rho}$ such that $\rho(F)=\rho(G)$ (note that here $\rho$ is treated as a functional on $D_{\rho}$ ). Imposing both requirements is very restrictive as demonstrated in Section 3. Note that condition (4.1) for all $F$ and $G$ leads to Choquet integrals, whereas condition (4.2) for all $F$ and $G$ leads to expected utility functions (von Neumann-Morgenstern Theorem); such observation leads to the dual theory of risk preference in Yaari (1987) and Schmeidler (1989). The intersection of both types of functionals is apparently only the mean. Elicitability only requires (4.2) for $F$ and $G$ such that $\rho(F)=\rho(G)$; this allows the Value-at-Risk (which does not belong to the class of expected utility functions) to still be generally elicitable.

Elicitability and comonotonic additivity both have their own justifications for applications, just like other properties considered in the literature, such as 
robustness and convexity. Depending on specific needs of the practical situation, some requirements may be more important than others. It appears that there is no risk measure that is recommendable in all situations; one should carefully choose the risk measure to use and know its limitations.

\section{Acknowledgement}

We thank Steve Kou and Xianhua Peng for helpful comments and suggestions on the paper. R. Wang acknowledges financial support from the Natural Sciences and Engineering Research Council of Canada (NSERC Grant No. 435844).

\section{References}

Acerbi, C. (2002). Spectral measures of risk: A coherent representation of subjective risk aversion. Journal of Banking and Finance, 26(7), 1505-1518.

Banerjee, A., Guo, X., and Wang, H. (2005). On the optimality fo conditional expectation as a Bregman predictor. IEEE Transactions on Information Theory, 51, 2664-2669.

Bellini, F. and Bignozzi, V. (2014). On elicitable risk measures. Quantitative Finance, to appear. Available at SSRN: http://ssrn.com/abstract=2334746, version Dec 2013.

Delbaen, F., Bellini, F., Bignozzi, V., and Ziegel, J. F. (2014). Risk measures with the CxLS property. Available at arXiv: http://arxiv.org/abs/1411.0426, version Nov 2014.

Denneberg, D. (1994). Non-additive measure and integral. Springer.

Embrechts, P., Puccetti, G., Rüschendorf, L., Wang, R. and Beleraj, A. (2014). An academic response to Basel 3.5. Risks, 2(1), 25-48. 
Gneiting, T. (2011). Making and evaluating point forecasts. Journal of the American Statistical Association, 106, 746-762.

Huber, P. J. and Ronchetti E. M. (2009). Robust statistics. Second ed., Wiley Series in Probability and Statistics. Wiley, New Jersey.

Kou, S. and Peng, X. (2014). On the measurement of economic tail risk. Available at arXiv: http://arxiv.org/abs/1401.4787, version Feb 2014.

Kusuoka, S. (2001). On law invariant coherent risk measures. Advances in Mathematical Economics, 3, 83-95.

Lambert, N. (2012). Elicitation and evaluation of statistical functionals. Available at: http://www.stanford.edu/ nlambert/papers/elicitation.pdf, version Apr 2012.

Osband, K. H. (1985). Providing incentives for better cost forecasting. Ph.D. thesis, University of California, Berkeley.

Savage, L. J. (1971). Elicitation of personal probabilities and expectations. Journal of the American Statistical Association, 66, 783-801.

Schmeidler, D. (1989). Subject probability and expected utility without additivity. Econometrica, 57(3), 571-587.

Steinwart, I., Pasin, C., Williamson, R. and Zhang, S. (2014). Elicitation and identification of properties. JMLR: Workshop and Conference Proceedings, 35, $1-45$.

Thomson, W. (1979). Eliciting production possibilities from a well-informed manager. Journal of Economic Theory, 20(3), 360-380.

Wang, S. S., Young, V. R. and Panjer, H. H. (1997). Axiomatic characterization of insurance prices. Insurance: Mathematics and Economics, 21(2), 173-183. 
Yaari, M. E. (1987). The dual theory of choice under risk. Econometrica, 55(1), $95-115$.

Weber, S. (2006). Distribution-invariant risk measures, information, and dynamic consistency. Mathematical Finance, 16, 419-441.

Ziegel, J. (2015). Coherence and elicitability. Mathematical Finance, to appear. DOI: $10.1111 / \operatorname{mafi} .12080$. 